\title{
Air pollution potential hot zone evaluation model based on environmental sensing IoT
}

\author{
Chi-Chieh Peng ${ }^{\mathrm{a}}$, Tzai-Hung Wen ${ }^{\mathrm{b}, *}$ \\ a (National Taiwan University) Chi-Chieh Peng,d07228002@ntu.edu.tw \\ b (National Taiwan University) Tzai-HungWen,wenthung@ntu.edu.tw \\ * Corresponding author
}

Keywords: IoT, Micro-Sensor, Air Pollution, PM2.5, Air Quality, Spatial Analysis, Environment Analysis

\begin{abstract}
:
PM2.5 is invisible to the eye, but it is a threat to human health. In order to monitor the small-scale environment status, Taiwan's Environmental Protection Administration (EPA) have deployed more than 2,500 environmental IoT Sensor in 13 cities (Figure 1) to monitor air quality. These sensors are mainly deployed in industrial areas, gather minute-byminute environmental data on PM2.5, temperature, and humid, etc., try to evaluate local pollution hot zone in each area.

Mainstream air quality sensing relies on high-precision large-scale stations, but it is difficult to collect local air quality information. In fact, Factories, temples or local air pollution emission behaviour can strongly affect the air quality, and some emissions have already exceeded acceptable limits. However, emissions disperse quickly with the wind in all directions, cause the environment changes greatly in a very short time. As a result, it is difficult to determine the source of emissions, which is a serious problem for environmental protection administration.
\end{abstract}
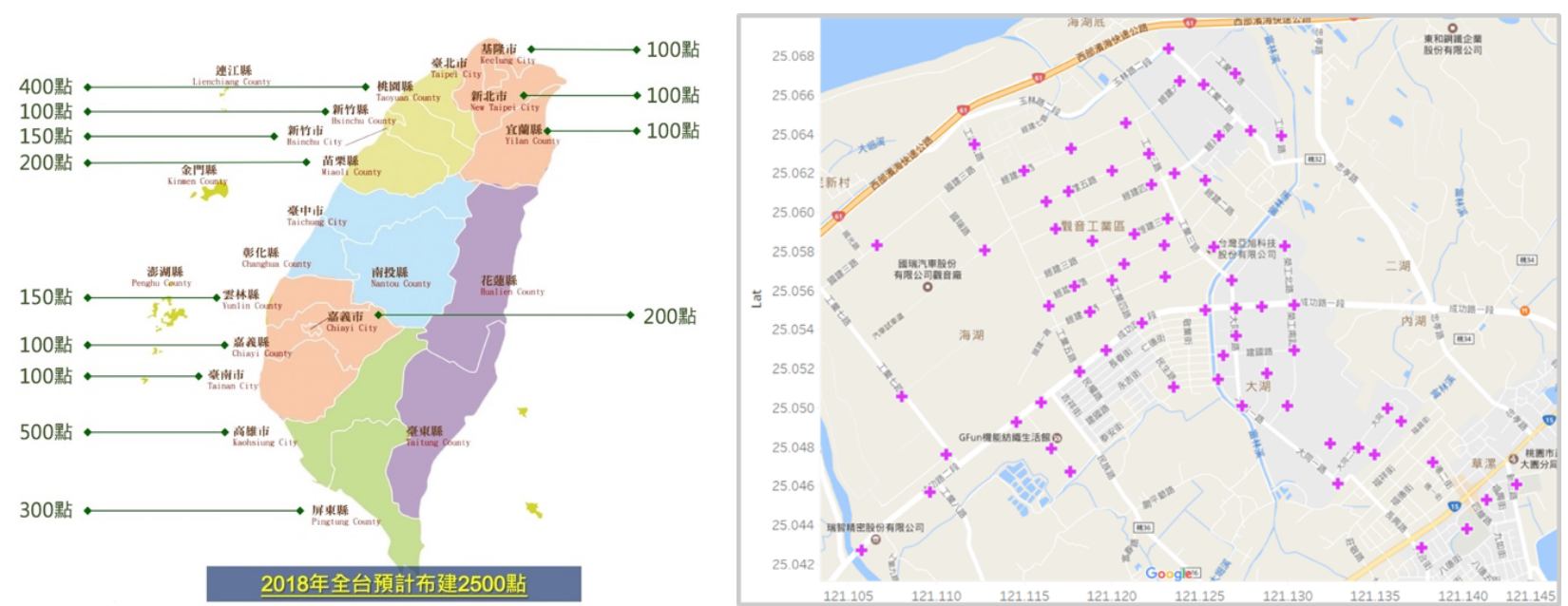

Figure 1. (left)Number of IoT deployed in 13 cities in Taiwan, (right) IoT deployment location in the industrial area

By environmental IoT sensors, this study collects local air quality information at a small scale (currently IoT installed at intervals of 300 meters), which enhance the air pollution monitor capabilities. Based on the detailed environmental information, this study established an air pollution potential hot zone evaluation model, detect the local suspicious pollution area through data variability, and calculate the grid-based area pollution scores, provide relevant information for EPA to strengthen pollution traceability.

Air pollution potential hot zone evaluation model estimate pollution scores through environment factors, such as PM2.5 concentration, wind, degree of data variation, we spread virtual circles every 300 meters in the study area, accumulate the pollution sensing value, and integrate time and spatial characteristics of the pollution, establish a fully automatic pollution analysis model, systematically mark the air pollution potential hot zone, and these hot zones information will deliver to EPA.

Taking Figure 2 as an example, if there is an abnormally emission event occurs at the potential pollution IoT (purple cross), and the range of influence covers the potential areas A, B, C, and D, will be affected. Accumulate all these patterns, we can infer the potential pollution hot zones automatically, distinguish normal and abnormal circumstances, and estimated as a polluted hot zone. 

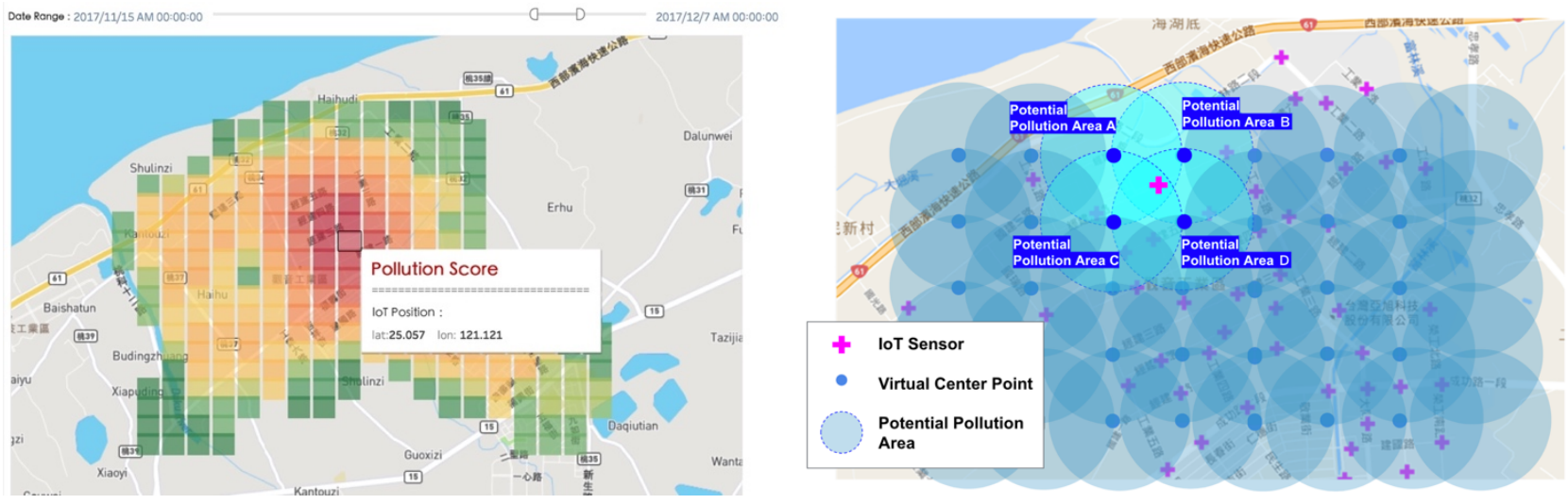

Figure 2. (left) Pollution scores will be automatically calculated in each IoT deployment area, (right) virtual centre point and its circle range

This study also implemented air quality cloud-based GIS system (Figure 3), and the potential hot zone information is built as a data layers, this system also combines the air pollution real-time sensing data, geographic information, time information, wind direction data, wind speed data, etc. The combined data are used to create data visualization, which enables the reconstruction of circumstances of the air pollution severity at a specific time range, which can assist environment officials to trace excessive emissions evidence.

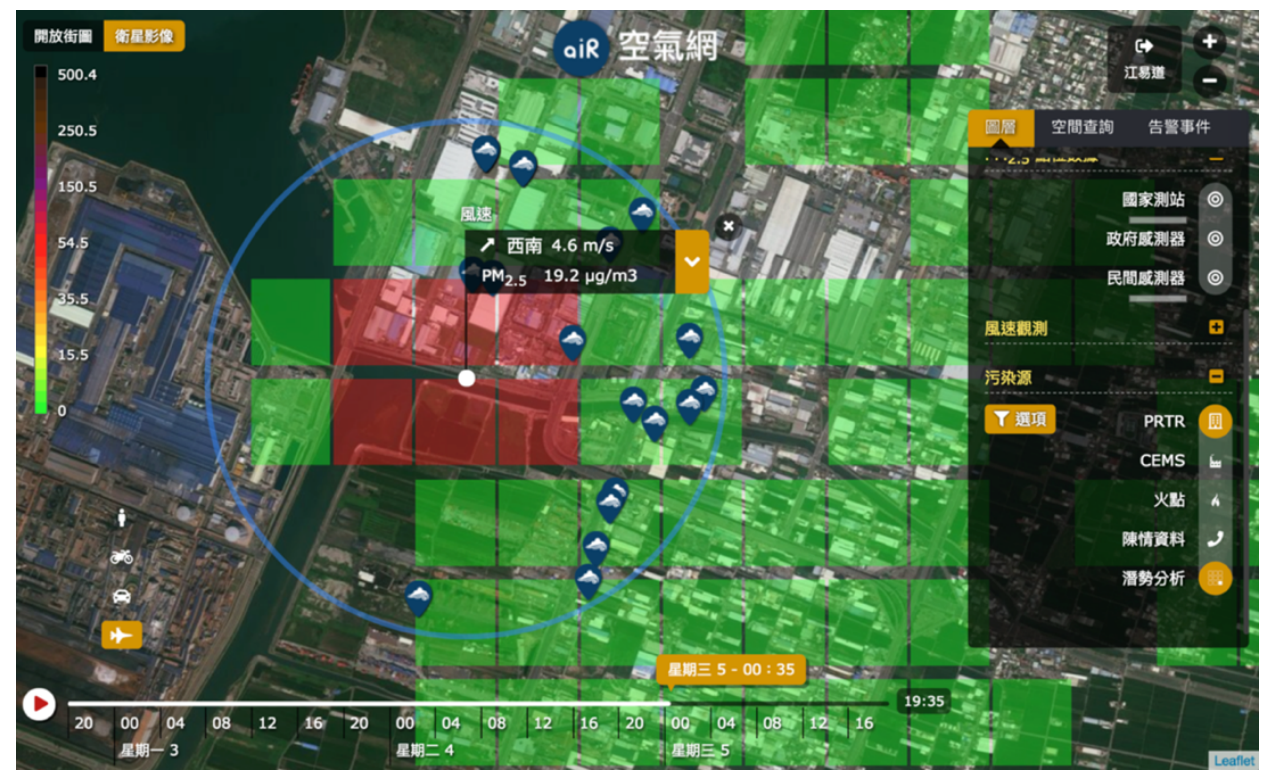

Figure 3. Web GIS interface display the air pollution hot zone information, the red grid is a more serious polluted hot zone than the green grid.

By environmental IoT Sensor data, this study proposed an air pollution potential hot zone evaluation model, which help EPA to find the local abnormal pollution areas. Therefore, environmental analysts can reference as policy recommendations, such as conduct environment inspections on certain days of the week, or certain time of the day, increasing the probability of catching serious emissions behaviour, and strengthen the environmental enforcement capacity. 